\title{
Satisfação com o Internato Médico em Portugal
}

\section{Medical Residency' Satisfaction in Portugal}

\author{
Miguel BIGOTTE VIEIRA $\triangleleft^{* 1,2}$, Pedro GODINHO*2,3, Nuno GAIBINO ${ }^{2,4}$, Raquel DIAS ${ }^{2,5}$, Agostinho SOUSA ${ }^{2,6}$, \\ Inês MADANELO ${ }^{2,7}$, Francisco RIBEIRO-MOURÃO 2,8 , Mariana BRANDÃO ${ }^{2,9}$, Sónia DUARTE ${ }^{2,10}$, Tiago MEIRINHOS M $^{2,11}$, \\ Ana Luísa CATARINO ${ }^{2}$, Cláudio ESPÍRITO SANTO ${ }^{2,12}$, Ricardo CAIADO ${ }^{2,13}$, Ricardo MARQUES ',14, \\ Ana Gonçalves FERREIRA ${ }^{2,15}$, Carlos RAMALHEIRA ${ }^{2,16}$, João Valente JORGE ${ }^{17}$, Marta LOSADA ${ }^{18,19}$, Mário SANTOS ${ }^{19,21}$, \\ Edson OLIVEIRA ${ }^{19,20}$, João Paulo FARIAS ${ }^{19,20}$, José Manuel SILVA ${ }^{22}$ \\ Acta Med Port 2016 Dec;29(12):839-853 - http://dx.doi.org/10.20344/amp.8406
}

\section{RESUMO}

Introdução: Nos últimos anos, o contexto global da formação médica, e em particular do Internato Médico em Portugal, sofreu profundas alterações. O presente estudo teve como objetivo avaliar e caracterizar a satisfação dos médicos internos com a realização do Internato Médico em Portugal e identificar aspetos passíveis de melhoria.

Material e Métodos: Foi utilizado como modelo de inquérito o questionário Postgraduate Hospital Educational Environment Measure desenvolvido no Reino Unido e dirigido a médicos internos, o qual foi traduzido e adaptado à realidade portuguesa. O questionário esteve disponível online durante os meses de abril e maio de 2016.

Resultados: Foram obtidas 3456 respostas, correspondendo a uma taxa de resposta de $35 \%$. Endocrinologia/Nutrição, Cardiologia, Anestesiologia, Medicina Geral e Familiar e Gastrenterologia foram as especialidades nas quais o grau de satisfação foi mais elevado, enquanto que Medicina Legal, Oncologia Médica, Medicina Interna, Cirurgia Geral e Pneumologia apresentaram o grau de satisfação mais baixo.

Discussão: O presente estudo apresenta uma elevada taxa de resposta comparativamente com estudos prévios. A nível nacional, no global, os médicos internos apresentaram níveis elevados de satisfação, destacando-se marcadas assimetrias de acordo com o ano de especialidade, região, tipologia de instituição e de especialidade.

Conclusão: Os resultados deste inquérito poderão constituir uma ferramenta de apoio à implementação de medidas de âmbito local e nacional relacionadas com o Internato Médico, sendo desejável a realização regular de inquéritos de satisfação aos médico internos. Palavras-chave: Avaliação Educacional; Educação Médica Pós-Graduada; Inquéritos e Questionários; Internato Médico; Portugal; Satisfação Pessoal.

\section{ABSTRACT}

Introduction: In the last years, the global context of medical education and Medical Residency programs in Portugal suffered substantial changes. The primary objective of this study was to evaluate and characterize medical residents' satisfaction with medical residency programs in Portugal and to identify features that could be improved.

Material and Methods: We utilized as model the survey Postgraduate Hospital Educational Environment Measure that has been developed in the United Kingdom and is specifically targeted to medical residents. The survey was translated and adapted to the Portuguese reality. The survey was available online during April and May of 2016.

Results: A total of 3456 responses were obtained, corresponding to a response rate of $35 \%$. Endocrinology/Nutrition, Cardiology, Anesthesiology, Family Physician and Gastroenterology were the specialties in which the degree of satisfaction was higher, while Forensic Medicine, Medical Oncology, Internal Medicine, General Surgery and Pneumology showed the lowest level of satisfaction.

\footnotetext{
* Co-primeiros autores.

1. Serviço de Nefrologia e Transplantação Renal. Centro Hospitalar Lisboa Norte. Lisboa. Portugal.

2. Conselho Nacional do Médico Interno. Ordem dos Médicos. Lisboa. Portugal.

3. Serviço de Anestesiologia. Centro Hospitalar de Leiria. Leiria. Portugal.

4. Serviço de Medicina I. Centro Hospitalar Lisboa Norte. Lisboa. Portugal.

5. Serviço de Imunohemoterapia. Hospital de Braga. Braga. Portugal.

6. Unidade Local de Saúde da Guarda. Guarda. Portugal.

7. Unidade de Cuidados de Saúde Personalizados de Vouzela. Vouzela. Portugal.

8. Serviço de Pediatria. Unidade Local de Saúde do Alto Minho. Viana do Castelo. Portugal.

9. Serviço de Oncologia Médica. Instituto Português de Oncologia do Porto Francisco Gentil. Porto. Portugal.

10. Serviço de Anestesiologia. Centro Hospitalar do Porto. Porto. Portugal.

11. Serviço de Reumatologia, Centro Hospitalar do Baixo Vouga. Aveiro. Portugal.

12. Medicina Geral e Familiar, Unidade de Saúde Familiar Buarcos. Buarcos. Portugal.

13. Serviço de Otorrinolaringologia. Centro Hospitalar e Universitário de Coimbra. Coimbra. Portugal.

14. Serviço de Medicina. Centro Hospitalar Universitário de Coimbra. Coimbra. Portugal.

15. Serviço de Endocrinologia. Hospital Garcia de Orta. Almada. Portugal.

16. Serviço de Medicina. Hospital Prof. Doutor Fernando Fonseca. Amadora. Portugal.

17. Serviço de Anestesiologia. Centro Hospitalar Lisboa Norte. Lisboa. Portugal.

18. Unidade de Saúde Pública. Unidade Local de Saúde do Alto Minho. Viana do Castelo. Portugal.

19. Conselho Nacional do Internato Médico. Administração Central dos Serviços de Saúde. Lisboa. Portugal.

20. Serviço de Neurocirurgia. Centro Hospitalar Lisboa Norte. Lisboa. Portugal.

21. Unidade de Saúde Pública. Unidade Local de Saúde do Litoral Alentejano. Santiago do Cacém. Portugal.

22. Conselho Nacional Executivo. Ordem dos Médicos. Lisboa. Portugal.

$\triangle$ Autor correspondente: Miguel Bigotte Vieira. mbigottevieira@gmail.com

Recebido: 01 de novembro de 2016 - Aceite: 01 de dezembro de 2016 | Copyright $\odot$ Ordem dos Médicos 2016
} 
Discussion: This study presented a high response rate when compared to previous studies. Portuguese medical residents presented high levels of satisfaction. Depending on year of medical residency, region, type of specialty and type of hospital marked asymmetries were noticed.

Conclusion: The survey's results should constitute in the future a support tool for the implementation of local and national measures relating to the medical residency. It is advisable to regularly conduct satisfaction surveys to medical residents.

Keywords: Education, Medical, Graduate; Educational Measurement; Internship and Residency; Personal Satisfaction; Portugal.

\section{INTRODUÇÃo}

Nos últimos anos, o contexto global da formação médica, e em particular, do Internato Médico em Portugal, sofreu profundas alterações, existindo vários fatores a contribuir para esta mudança., ${ }^{1,2}$ Desde 1995, o número de estudantes admitidos no curso de Medicina em Portugal aumentou cerca de $400 \%$, o que coloca Portugal em primeiro lugar entre os países da Organização de Cooperação e de Desenvolvimento Económico (OCDE) no que se refere ao aumento percentual do número de estudantes formados em Medicina. ${ }^{1}$ Em 2014, de acordo com dados da OCDE, Portugal constituía o quarto país membro com maior número de médicos, apresentando 4,3 médicos por cada 1000 habitantes. ${ }^{3}$ A demografia médica em Portugal caracteriza-se pela existência de assimetrias na distribuição de médicos pelas diferentes especialidades e entre as regiões rurais e urbanas. ${ }^{4} \mathrm{Um}$ estudo realizado em 2013 sobre este tema concluiu existir uma tendência de aumento do pessoal médico ao longo das últimas três décadas, traduzindo-se este aumento num desajustamento entre a produção de médicos especialistas e as respetivas necessidades nacionais, perspetivando-se deste modo um número excedentário de especialistas não absorvidos pelo sistema. Este desajustamento não é, contudo, homogéneo, sendo superior em algumas especialidades médicas. ${ }^{5} \mathrm{~A}$ legislação que regulamenta o Internato Médico em Portugal foi revista em 2015. 6,7

Conciliar trabalho e aprendizagem em ambiente clínico constitui uma tarefa exigente para os médicos internos. ${ }^{8}$ A existência de uma cultura institucional de aprendizagem clínica é fundamental na formação de médicos competentes. ${ }^{9}$ Doentes acompanhados por médicos insatisfeitos e desmotivados apresentam maior grau de insatisfação com os cuidados médicos recebidos e piores indicadores de saúde..$^{10,11}$ De salientar ainda que médicos insatisfeitos apresentam maior taxa de absentismo laboral e risco de burnout. ${ }^{12}$ Neste contexto, e tendo em consideração o expectável aumento da competitividade entre os médicos internos, é importante que seja avaliado o panorama geral do Internato Médico em Portugal. Atualmente, em Portugal, o Internato Médico é organizado num primeiro ano de formação geral, denominado 'Ano Comum', seguido de um período de formação com duração entre quatro e seis anos, denominado 'Internato de Formação Específica', de acordo com a especialidade escolhida pelo médico interno.

A nível internacional, a realização de inquéritos de satisfação aos médicos internos é prática corrente. ${ }^{13-19}$ Roff et al desenvolveram um questionário intitulado "The Postgraduate Hospital Education Environment Measure (PHEEM)", no Reino Unido, com esta finalidade. Desde 2004 este questionário foi traduzido e aplicado em instituições de diversos países. ${ }^{8,20-31} \mathrm{Em}$ Portugal, não está implementada a realização regular de inquéritos de satisfação aos médicos internos. Encontra-se publicado na literatura um reduzido número de inquéritos realizados no nosso país, destacando-se a escassa informação disponível sobre os fatores que caracterizam a satisfação dos Médicos Internos.

O presente estudo teve como objetivo principal avaliar de forma metódica a satisfação dos médicos internos com a realização do Internato Médico em Portugal. Pretendemos como objetivos secundários caracterizar a satisfação dos médicos internos com a realização do Internato Médico em diferentes especialidades, em diferentes regiões e em diferentes tipologias hospitalares de instituição de formação, bem como identificar aspetos passíveis de melhoria.

\section{MATERIAL E MÉTODOS Inquérito}

Foi utilizado como modelo de inquérito (Tabela 1) um questionário desenvolvido no Reino Unido especialmente dirigido a médicos internos (Postgraduate Hospital Educational Environment Measure - PHEEM), o qual foi validado e utilizado em diferentes países.,20-31 Este questionário é constituído por 40 afirmações, sendo respondido em poucos minutos. A tradução e adaptação do questionário foram realizadas por médico interno com certificado Certificate of Proficiency in English emitido por Cambridge English Language Assessment, tendo sido revista por um grupo de trabalho composto por médicos de diferentes especialidades, também fluentes em inglês. Foi solicitada autorização por escrito aos autores do questionário original para a tradução, adaptação e aplicação do questionário a nível nacional em Portugal. Na adaptação à realidade portuguesa optámos por incluir uma afirmação referente à satisfação em geral com a Direção/Coordenação do Internato Médico (afirmação 41) e duas afirmações sobre a satisfação com o orientador de formação em particular (afirmações 42 e 43). Não foi realizada validação estatística da tradução e adaptação do questionário para a população portuguesa.

\section{Aplicação do inquérito}

A realização do inquérito teve 0 apoio do Conselho Nacional do Médico Interno (CNMI) e do Conselho Nacional Executivo (CNE) da Ordem dos Médicos, bem como do Conselho Nacional do Internato Médico (CNIM), órgão consultivo da Administração Central dos Serviços de Saúde (ACSS).

O questionário foi de resposta anónima e participação voluntária, tendo estado disponível online durante os 
Tabela 1 - Modelo de Inquérito de Satisfação aos Médicos Internos

\begin{tabular}{ll} 
Qual a sua área de especialização? & $\begin{array}{l}\text { (Listagem de Especialidades) } \\
\text { Qual a sua instituição de colocação? }\end{array}$ \\
$\begin{array}{l}\text { Caso seja Interno de Formação Específica em que ano da sua } \\
\text { formação se encontra? }\end{array}$ & $\begin{array}{l}\text { (Listagem de anos de Formação Específica) } \\
\text { Qual o seu sexo? }\end{array}$ \\
\hline
\end{tabular}
As seguintes questões referem-se à sua instituição de colocação, independentemente do local onde se encontra a estagiar
atualmente. Leia por favor cada frase e indique de que modo concorda ou discorda com a mesma.

1 - Eu tenho um contrato de trabalho que estabelece informação sobre horas de trabalho.

2 - Os meus responsáveis de estágio definem expectativas claras.

3 - Eu tenho tempo de estudo definido no meu posto de trabalho.

4 - Eu tive um programa informativo introdutório.

5 - Eu tenho um nível de responsabilidade adequado no meu posto de trabalho.

6 - Eu tenho boa supervisão médica a todas as horas.

7 - Ocorre discriminação racial no meu posto de trabalho.

8 - Eu tenho que realizar tarefas inapropriadas.

9 - Existe um manual informativo para os médicos internos.

10 - Os meus responsáveis de estágio têm boas capacidades de comunicação.

11 - Eu sou contactado telefonicamente de modo inapropriado.

12 - Eu tenho oportunidade de participar ativamente em eventos formativos.

13 - Ocorre discriminação sexual no meu posto de trabalho.

14 - Existem protocolos de atuação bem definidos no meu posto de trabalho.

15 - Os meus responsáveis de estágio são entusiásticos.

16 - Eu tenho boa colaboração com outros médicos no meu nível de formação.

17 - O meu horário está de acordo com o Regulamento do Internato Médico.

18 - Eu tenho oportunidade de acompanhar a continuidade do tratamento/intervenção.

19 - Eu tenho acesso adequado a aconselhamento sobre a carreira.

20 - Esta instituição de colocação tem alojamento de qualidade para Internos, especialmente quando estão de urgência.

21 - Existe acesso a um programa educacional relevante para as minhas necessidades.

22 - Eu recebo feedback regular dos médicos especialistas.

23 - Os meus responsáveis de estágio estão bem organizados.

24 - Eu sinto-me seguro fisicamente na instituição de colocação.

25 - Existe uma cultura de não culpabilização no meu posto de trabalho.

26 - Existem serviços de restauração adequados quando estou de Urgência.

27 - Eu tenho oportunidades de aprendizagem médica suficientes para as minhas necessidades.

28 - Os meus responsáveis de estágio possuem boas capacidades formativas.

29 - Eu sinto-me membro de uma equipa ao trabalhar neste local.

30 - Eu tenho oportunidade de praticar procedimentos adequados ao meu nível de formação.

31 - Os meus responsáveis de estágio são acessíveis.

32 - A minha carga de trabalho neste emprego é adequada.

33 - Os médicos especialistas utilizam as oportunidades de aprendizagem eficazmente.

34 - O treino obtido no meu posto de trabalho faz-me sentir preparado para ser médico especialista.

35 - Os meus responsáveis de estágio são bons mentores.

36 - Eu obtenho grande satisfação pessoal do meu trabalho atual.

37 - Os meus responsáveis de estágio encorajam-me a adquirir conhecimentos de modo autónomo.

38 - Existem boas oportunidades de aconselhamento para médicos internos que não consigam terminar o seu internato.

39 - Os meus responsáveis de estágio providenciam-me bom feedback sobre os meus pontos fortes e pontos fracos.

40 - Os meus responsáveis de estágio promovem um ambiente de respeito mútuo.

41 - Estou satisfeito com a Direção/Coordenação do Internato Médico da minha instituição de colocação.

42 - O meu orientador de formação possui boas capacidades formativas.

43 - O meu orientador de formação tem conhecimentos científicos sólidos para proporcionar uma boa formação.

(Para cada uma das 43 afirmações, foi pedido aos médicos internos que escolhessem uma das seguintes cinco opções: Concordo Totalmente; Concordo; Indiferente; Discordo; Discordo Totalmente) 
meses de abril e maio de 2016, correspondendo a um estudo transversal. Foi realizada divulgação do inquérito através de correio eletrónico enviado pelas Direções/Coordenações do Internato Médico e pelas Comissões de Internos de cada Instituição aos médicos internos, bem como através das redes sociais, nomeadamente páginas de grupos de médicos internos. No cálculo das taxas de resposta, foram considerados os dados disponibilizados pela ACSS relativamente ao número de médicos internos existente à data de realização do inquérito.

Para a realização da análise dos resultados obtidos foi utilizada a metodologia proposta para o questionário original PHEEM. Este questionário é constituído por 40 afirmações que abrangem diferentes aspetos relevantes para o ambiente educativo dos médicos internos em determinada instituição. É pedido aos médicos internos que respondam

Tabela 2 - Tipologia de Instituição Hospitalar (Portaria n82/2014) a cada afirmação com uma escala de Likert de 5 pontos variando de "concordo totalmente" a "discordo totalmente". As respostas são pontuadas de 0 a 4: 4 'concordo totalmente'; 3 'concordo', 2 'indiferente', 1 'discordo', 0 'discordo totalmente'. De realçar que quatro das afirmações são de caráter negativo, pelo que são pontuadas de modo inverso (0 'concordo totalmente', 1 'concordo', 2 'indiferente', 3 'discordo', 4 'discordo totalmente'). Este questionário tem pontuação máxima global de 160, o que corresponderá ao ambiente educativo ideal proporcionado pela instituição, percecionado pelos médicos internos. De acordo com os autores do questionário poderá ser considerada a seguinte grelha de avaliação da pontuação global obtida: 0 a 40, muito fraco; 41 a 80 , muitos problemas; 81 a 120 , mais positivo do que negativo mas com potencial de melhoria; 121 a 160 , excelente. Além da pontuação global e individual

\begin{tabular}{|c|c|}
\hline Tipologia I & Tipologia II \\
\hline Centro Hospitalar Barreiro/Montijo & Centro Hospitalar de Lisboa Ocidental \\
\hline Centro Hospitalar Cova da Beira & Centro Hospitalar do Algarve \\
\hline Centro Hospitalar de Leiria & Centro Hospitalar Tondela-Viseu \\
\hline Centro Hospitalar de Setúbal & Centro Hospitalar Trás-os-Montes e Alto Douro \\
\hline Centro Hospitalar do Alto Ave & Centro Hospitalar Vila Nova de Gaia/Espinho \\
\hline Centro Hospitalar do Baixo Vouga & Hospital de Braga \\
\hline Centro Hospitalar do Médio Ave & Hospital Espírito Santo de Évora \\
\hline Centro Hospitalar do Oeste & Hospital Garcia de Orta \\
\hline \multicolumn{2}{|l|}{ Centro Hospitalar de Entre Douro e Vouga } \\
\hline \multicolumn{2}{|l|}{ Centro Hospitalar Médio Tejo } \\
\hline \multicolumn{2}{|l|}{ Centro Hospitalar Póvoa de Varzim/Vila do Conde } \\
\hline \multicolumn{2}{|l|}{ Centro Hospitalar Tâmega e Sousa } \\
\hline \multicolumn{2}{|l|}{ Hospital de Cascais } \\
\hline \multicolumn{2}{|l|}{ Hospital de Loures } \\
\hline \multicolumn{2}{|l|}{ Hospital de Vila Franca de Xira } \\
\hline \multicolumn{2}{|l|}{ Hospital Distrital da Figueira da Foz } \\
\hline \multicolumn{2}{|l|}{ Hospital Distrital de Santarém } \\
\hline \multicolumn{2}{|l|}{ Hospital Prof. Doutor Fernando da Fonseca } \\
\hline \multicolumn{2}{|l|}{ Hospital Santa Maria Maior } \\
\hline \multicolumn{2}{|l|}{ Unidade Local de Saúde da Guarda } \\
\hline \multicolumn{2}{|l|}{ Unidade Local de Saúde de Castelo Branco } \\
\hline \multicolumn{2}{|l|}{ Unidade Local de Saúde de Matosinhos } \\
\hline \multicolumn{2}{|l|}{ Unidade Local de Saúde do Alto Minho } \\
\hline \multicolumn{2}{|l|}{ Unidade Local de Saúde do Baixo Alentejo } \\
\hline \multicolumn{2}{|l|}{ Unidade Local de Saúde do Litoral Alentejano } \\
\hline \multicolumn{2}{|l|}{ Unidade Local de Saúde do Nordeste } \\
\hline \multicolumn{2}{|l|}{ Unidade Local de Saúde Norte Alentejo } \\
\hline Tipologia III & Tipologia IV \\
\hline Centro Hospitalar de Lisboa Central & Centro de Medicina de Reabilitação da Região do Centro - Rovisco Pais \\
\hline Centro Hospitalar de São João & Centro de Medicina Física de Reabilitação do Sul \\
\hline Centro Hospitalar e Universitário de Coimbra & Centro de Reabilitação do Norte \\
\hline Centro Hospitalar Lisboa Norte & Centro Hospitalar Psiquiátrico de Lisboa \\
\hline \multirow[t]{4}{*}{ Centro Hospitalar do Porto } & Hospital Magalhães de Lemos \\
\hline & Instituto Português de Oncologia de Coimbra \\
\hline & Instituto Português de Oncologia de Lisboa \\
\hline & Instituto Português de Oncologia do Porto \\
\hline
\end{tabular}


das 40 afirmações, os autores do PHEEM, consideram na análise dos resultados, 3 categorias de afirmações destinadas a avaliar diferentes aspectos: Ensino (afirmações 2, 3, $6,10,12,15,21,22,23,27,28,31,33,37,39)$, Autonomia (afirmações 1, 4, 5, 8, 9, 11, 14, 17, 18, 29, 30, 32, 34, 40) e Apoio (afirmações 7, 13, 16, 19, 20, 24, 25, 26, 35, 36, 38). Dado o questionário original ser destinado a especialidades hospitalares, em duas afirmações do presente questionário foi incluída a opção 'Não Aplicável' (afirmação 20 e 26). De modo a não condicionar o conteúdo das respostas nem diminuir o número de participantes, as 43 afirmações do questionário não tiveram caráter obrigatório.

Foram realizadas análises por subgrupo quanto ao sexo, ano de Formação Específica, região (de acordo com as Comissões Regionais de Internato Médico), tipologia de instituição hospitalar (de acordo com a tipologia de cada unidade hospitalar definida na Portaria n82/2014, apresentada na Tabela 2), especialidade e grupo de especialidade. ${ }^{32}$ Entende-se por 'grupo de especialidade' as seguintes categorias: Especialidades Médicas (Anestesiologia, Cardiologia, Cardiologia Pediátrica, Dermatovenereologia, Doenças Infeciosas, Endocrinologia/Nutrição, Farmacologia Clínica, Gastroenterologia, Genética Médica, Hematologia Clínica, Imunoalergologia, Imunohemoterapia, Medicina Desportiva, Medicina Física e Reabilitação, Medicina Interna, Medicina Legal, Medicina do trabalho, Nefrologia, Neurologia, Oncologia Médica, Pediatria, Pneumologia, Psiquiatria, Psiquiatria da Infância e da adolescência, Reumatologia, Radioncologia); Especialidades Cirúrgicas (Angiologia/Cirurgia Vascular, Cirurgia Cardíaca, Cirurgia Geral, Cirurgia Maxilo-facial, Cirurgia Pediátrica, Cirurgia Plástica, Cirurgia Torácica, Estomatologia, Ginecologia/Obstetrícia, Neurocirurgia, Oftalmologia, Ortopedia, Otorrinolaringologia, Urologia), Meios Complementares de Diagnóstico e Terapêutica (Anatomia Patológica, Patologia Clínica, Medicina Nuclear, Radiologia, Neurorradiologia); Medicina Geral e Familiar e Saúde Pública.

\section{Métodos estatísticos}

Foi realizada uma análise descritiva das variáveis. As variáveis categóricas foram descritas através de frequências absolutas e relativas expressas sob a forma de percentagem (\%), e as variáveis contínuas através da média e desvio-padrão. Foram realizadas comparações estatísticas entre os valores médios com recurso ao teste $t$ para amostras independentes e análise de variância (ANOVA). Valores de valor $p$ inferiores a 0,05 (nível de significância) foram considerados estatisticamente significativos. A análise estatística foi realizada com recurso ao software SPSS versão 21.0 .

\section{RESULTADOS}

Dos 9774 médicos internos a realizar o Internato Médico à data de realização do inquérito, foram obtidas 3456 (35\%) respostas, das quais $68 \%$ foram de médicos internos do sexo feminino e $32 \%$ do sexo masculino. Relativamente à duração de preenchimento do questionário, 31\% dos médicos internos demoraram um período de tempo inferior a 5 minutos, $52 \%$ demoraram entre 5 e 10 minutos e $17 \%$ demoraram mais de 10 minutos.

De acordo com o ano de Internato Médico, responderam $27 \%$ dos médicos internos do Ano Comum, 34\% dos do primeiro ano, $36 \%$ dos do segundo ano, $42 \%$ dos do terceiro ano, $41 \%$ dos do quarto ano, $35 \%$ dos do quinto ano e $29 \%$ dos do sexto ano.

De acordo com a região de formação, foram obtidas as seguintes taxas de resposta em cada região: $31 \%$ no Norte, $37 \%$ no Centro, 39\% em Lisboa e Vale do Tejo, 32\% no Alentejo, 36\% no Algarve, 25\% na Região Autónoma nos Açores e $27 \%$ na Região Autónoma da Madeira. Relativamente à distribuição por especialidades destacamos que 34 das 48 especialidades apresentaram taxa de resposta superior a $30 \%$ (Tabela 3 ).

$\mathrm{Na}$ Tabela 4 apresentam-se os itens ordenados de acordo com a pontuação média obtida e encontram-se assinaladas com '\#\#' as afirmações com pontuação invertida. A uma pontuação superior corresponde um maior número de respostas concordantes com a afirmação, enquanto a uma pontuação inferior corresponde um maior número de respostas discordantes com a afirmação, com exceção das quatro afirmações com pontuação invertida, nas quais ocorre o inverso. Nesta Tabela 4 encontram-se ainda assinaladas as respostas por sexo e por período de formação, nomeadamente Ano Comum e Internato de Formação Específica. Não houve diferença estatisticamente significativa entre sexos na pontuação global obtida (pontuação global média do sexo feminino, 91,3; pontuação global média do sexo masculino 93,0; valor $p=0,06$ ). Os médicos internos de Ano Comum obtiveram pontuação global mais elevada do que os médicos internos de Formação Específica, tendo esta diferença sido estatisticamente significativa (pontuação global média dos médicos Internos do Ano Comum, 98,0; pontuação global média dos médicos internos de Formação Específica 90,5, valor $p<0,01$ ).

Das 43 afirmações do questionário, identificaram-se seis cuja pontuação obtida foi a mais elevada (maior ou igual a 3,0 na escala de Likert). Destaca-se que os médicos internos, no global, tendem a considerar que não ocorre discriminação racial nem sexual no seu local de trabalho (afirmações com pontuação invertida), têm boa colaboração com os outros médicos no seu nível de formação, consideram que o seu orientador de formação tem conhecimentos sólidos para proporcionar uma boa formação e têm oportunidade de participar ativamente em eventos formativos.

No extremo oposto, as cinco afirmações cuja pontuação foi a mais baixa (menor ou igual a 1,6 na escala de Likert), revelam: os médicos internos tendem a considerar que não têm tempo de estudo definido no seu posto de trabalho; não existem boas oportunidades de aconselhamento para médicos internos que não consigam terminar o seu Internato Médico; a sua instituição de colocação não tem alojamento de qualidade para médicos internos, especialmente quando estão de urgência; inexistência de um manual 
Tabela 3 - Número de médicos internos por especialidade, número de respostas e taxa de resposta

\begin{tabular}{|c|c|c|c|}
\hline Especialidade & Número de Internos & Respostas obtidas (n) & Taxa de resposta (\%) \\
\hline Medicina Legal & 28 & 28 & 100,00 \\
\hline Cirurgia Cardíaca & 6 & 4 & 66,67 \\
\hline Cirurgia Torácica & 6 & 4 & 66,67 \\
\hline Cirurgia Pediátrica & 24 & 13 & 54,17 \\
\hline Saúde Pública & 153 & 82 & 53,59 \\
\hline Reumatologia & 51 & 26 & 50,98 \\
\hline Farmacologia Clínica & 2 & 1 & 50,00 \\
\hline Medicina Desportiva & 6 & 3 & 50,00 \\
\hline Endocrinologia/Nutrição & 74 & 34 & 45,95 \\
\hline Medicina Nuclear & 16 & 7 & 43,75 \\
\hline Psiquiatria da Infância e da Adolescência & 65 & 28 & 43,08 \\
\hline Medicina Geral e Familiar & 1939 & 835 & 43,06 \\
\hline Anestesiologia & 355 & 152 & 42,82 \\
\hline Pneumologia & 151 & 61 & 40,40 \\
\hline Neurologia & 130 & 52 & 40,00 \\
\hline Nefrologia & 102 & 39 & 38,24 \\
\hline Medicina do Trabalho & 22 & 8 & 36,36 \\
\hline Ginecologia/Obstetrícia & 259 & 94 & 36,29 \\
\hline Cardiologia & 149 & 54 & 36,24 \\
\hline Medicina Interna & 1067 & 385 & 36,08 \\
\hline Cirurgia Geral & 350 & 126 & 36,00 \\
\hline Psiquiatria & 297 & 92 & 30,98 \\
\hline Dermatovenerealogia & 48 & 17 & 35,42 \\
\hline Neurocirurgia & 54 & 19 & 35,19 \\
\hline Urologia & 67 & 23 & 34,33 \\
\hline Doenças Infeciosas & 84 & 28 & 33,33 \\
\hline Pediatria & 378 & 124 & 32,80 \\
\hline Oftalmologia & 101 & 33 & 32,67 \\
\hline Neurorradiologia & 37 & 12 & 32,43 \\
\hline Oncologia Médica & 171 & 55 & 32,16 \\
\hline Medicina Física e de Reabilitação & 102 & 32 & 31,37 \\
\hline Imunohemoterapia & 88 & 27 & 30,68 \\
\hline Imunoalergologia & 49 & 15 & 30,61 \\
\hline Estomatologia & 43 & 13 & 30,23 \\
\hline Otorrinolaringologia & 117 & 35 & 29,91 \\
\hline Ortopedia & 259 & 75 & 28,96 \\
\hline Cirurgia Maxilo-facial & 21 & 6 & 28,57 \\
\hline Cardiologia Pediátrica & 22 & 6 & 27,27 \\
\hline Ano Comum & 2147 & 584 & 27,20 \\
\hline Cirurgia Plástica, Reconstrutiva e Estética & 52 & 14 & 26,92 \\
\hline Gastrenterologia & 117 & 31 & 26,50 \\
\hline Radiologia & 138 & 36 & 26,09 \\
\hline Angiologia/Cirurgia Vascular & 48 & 12 & 25,00 \\
\hline Radioncologia & 40 & 9 & 22,50 \\
\hline Hematologia Clínica & 90 & 19 & 21,11 \\
\hline Patologia clínica & 113 & 23 & 20,35 \\
\hline Anatomia Patológica & 82 & 16 & 19,51 \\
\hline Genética Médica & 26 & 5 & 19,23 \\
\hline Cirurgia Cardiotorácica & 28 & 3 & 10,71 \\
\hline
\end{tabular}


informativo para os médicos internos; ausência de acesso adequado a aconselhamento sobre a carreira.

Analisando apenas as especialidades com número de respostas superior a 20 (Fig. 1), verifica-se que Endocrinologia/Nutrição, Cardiologia, Anestesiologia, Medicina Geral e Familiar e Gastrenterologia são as especialidades nas quais o grau de satisfação, avaliado pela pontuação global obtida na resposta ao questionário, foi mais elevado. As especialidades de Medicina Legal, Oncologia Médica, Medicina Interna, Cirurgia Geral e Pneumologia foram as que apresentaram grau de satisfação mais reduzido.

$\mathrm{Na}$ Tabela 5 encontra-se assinalada a pontuação obtida em cada uma das três afirmações incluídas na adaptação do questionário à realidade portuguesa bem como a pontuação obtida em cada uma das três categorias do inquérito: Autonomia, Ensino e Apoio. Os médicos internos do Ano Comum constituem o grupo que apresenta grau de satisfação global mais elevado. Avaliando a evolução do grau de satisfação por ano de Internato de Formação Específica (Tabela 5B), verifica-se existir tendência para o seu decréscimo do primeiro ao quinto ano, aumentando no sexto ano. Entre os médicos Internos de Formação Específica, a ordenação em sentido decrescente do grau de satisfação por grupo de especialidade (Tabela 5C) foi: Medicina Geral e Familiar, Saúde Pública, Meios Complementares de Diagnóstico e Terapêutica; Especialidades Cirúrgicas; Especialidades Médicas. Na análise por regiões (Tabela $5 A$ ), verifica-se que a região na qual os médicos internos apresentam grau de satisfação mais elevado é a Madeira, seguida dos Açores, Alentejo, Centro, Norte, Lisboa e Vale do Tejo e Algarve, por ordem decrescente de grau de satisfação. Quanto à tipologia de instituição hospitalar (Tabela 5D) verifica-se que os médicos internos de hospitais de tipologia I (instituições com área de influência direta entre 75000 e 500000 habitantes, sem área de influência indireta) apresentam o grau de satisfação mais elevado, de entre as quatro tipologias de hospitais. Relativamente à satisfação com a Direção do Internato Médico, salienta-se que, os médicos internos que se encontram mais satisfeitos são os de hospitais de tipologia I bem como os médicos internos dos Açores e da Madeira.

\section{DISCUSSÃO}

Consideramos que a percentagem de respostas obtida a nível nacional (35\%) é satisfatória, considerando as taxas de resposta obtidas em estudos semelhantes. ${ }^{12}$ Ainda assim, acreditamos que a aplicação periódica do questionário e, em particular, a análise dos resultados obtidos para orientar a adoção de medidas que visem a melhoria do Internato Médico, poderão, no futuro, contribuir para um aumento da taxa de respostas. A distribuição das respostas entre sexos reflete a tendência verificada nos últimos anos do predomínio de estudantes do sexo feminino nas faculdades de Medicina a nível nacional. ${ }^{5}$ Realçamos que a taxa de resposta obtida, quando analisada quer de acordo com o ano de Internato Médico quer de acordo com a região de formação, apresenta um padrão próximo do que era expectável em termos de representatividade da amostra. De salientar o elevado número de especialidades com taxa de resposta elevada, sendo que em apenas 6 foi obtida uma taxa de resposta inferior a $25 \%$. A realização de questionário anónimo, e a opção pela não obrigatoriedade de resposta à totalidade das afirmações, permitiu respostas francas, evitando o condicionamento no seu preenchimento. De salientar que a pergunta que obteve menor número de respostas foi a que permitia a identificação do ano de Formação Específica, o que poderá estar relacionado com a importância da garantia de anonimato deste modelo de questionário. Estes aspetos favorecem a importância dos dados obtidos, pelo que consideramos que as respostas podem ser analisadas como ferramenta robusta na avaliação do Internato Médico em Portugal.

Destacamos que apesar de os médicos internos do sexo masculino terem apresentado um grau de satisfação superior aos médicos internos do sexo feminino, esta diferença não foi estatisticamente significativa. Coloca-se como hipótese para os médicos internos de Ano Comum terem obtido pontuação global mais elevada do que os médicos internos de Formação Específica o facto de o Ano Comum ser um período de formação no Internato Médico no qual o interno contacta com o doente enquanto médico pela primeira vez, mas ainda sem autonomia ou responsabilidade excessiva. ${ }^{7}$ Tal conduz a que o nível de exigência a que o médico interno se encontra sujeito, quer pelo próprio, quer pelos seus pares, seja menor. De salientar ainda a menor sobrecarga assistencial a que os médicos internos de Ano Comum se encontram sujeitos, devido ao seu menor grau de diferenciação, em comparação com os médicos internos de Formação Específica. Por último, o facto dos médicos internos de Ano Comum se encontrarem a realizar o Internato Médico há menos tempo e os estágios em cada serviço terem curta duração, poderá contribuir para que tenham uma menor perceção de alguns dos problemas a que os médicos internos de Formação Específica se encontram expostos.

Apresenta-se, como hipótese para a tendência detetada de diminuição do grau de satisfação ao longo do internato, a eventual progressiva tomada de consciência, durante o internato, da realidade existente na especialidade, instituição de formação e perspetiva futura de colocação no mercado de trabalho, as quais poderão não corresponder totalmente ao idealizado no momento da escolha. De realçar ainda que o aumento do grau de satisfação verificado no sexto ano deve ser interpretado com reserva, dado que o número de especialidades com programa de formação com 6 anos de duração é reduzido, correspondendo a especialidades cirúrgicas, e que a percentagem de respostas de médicos internos do sexto ano foi, entre os médicos internos de Formação Específica, a mais baixa.

Considerando os diferentes grupos de especialidades, as especialidades de MGF e Saúde Pública apresentaram maior grau de satisfação. Coloca-se como hipótese para este resultado a menor realização de trabalho de urgência, em particular urgência noturna e durante os fins de 


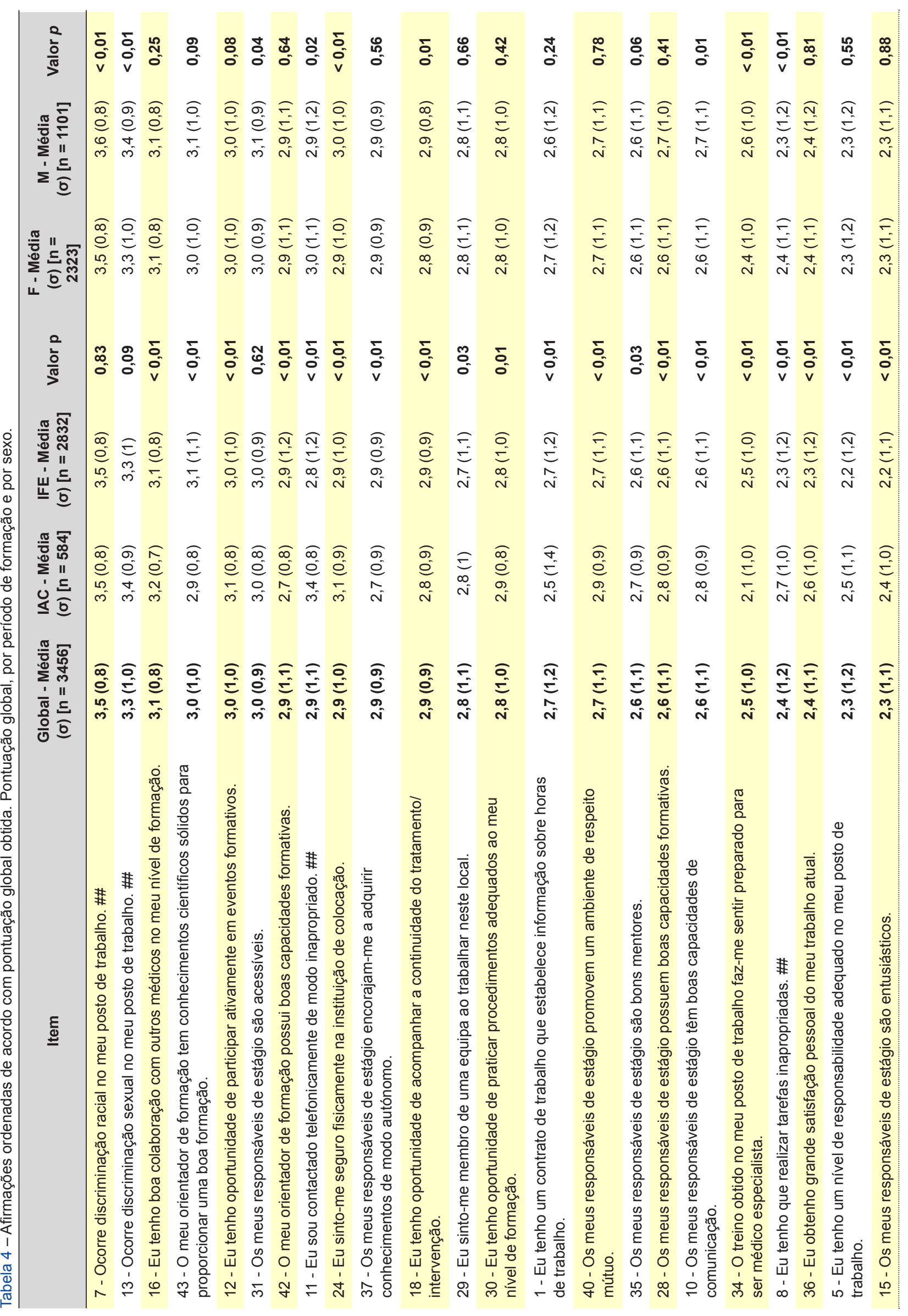

Tabela 4 continua na próxima página 


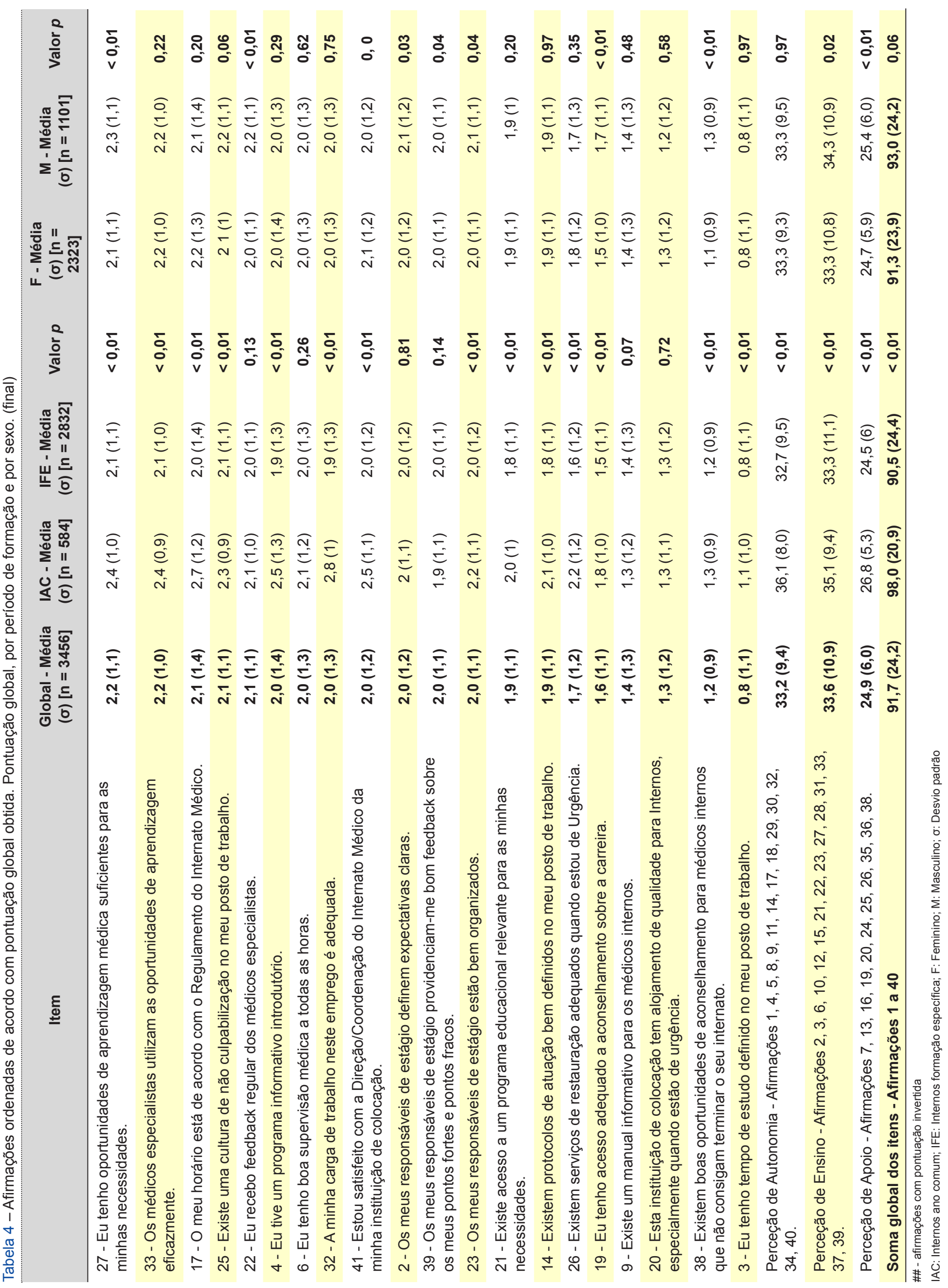


semana, fator de sobrecarga para os médicos internos nas especialidades hospitalares. De destacar o maior grau de satisfação dos médicos internos da região dos Açores e da Madeira, aspeto que poderá estar relacionado com as medidas de incentivo e apoio instituídas pelas respetivas regiões autónomas com o objetivo de fixação de médicos na região. ${ }^{33}$ De salientar ainda o menor grau de satisfação dos médicos internos da região do Algarve, região que apresen- tou nos últimos anos um maior número de vagas por preencher nos concursos destinados à colocação de recém-especialistas e que apresenta uma conhecida carência de médicos especialistas, fatores que poderão aumentar a sobrecarga de trabalho exigida aos médicos internos e contribuir para o menor grau de satisfação dos mesmos. ${ }^{34}$ Estes aspetos corroboram a importância da análise do panorama do Internato Médico nesta região.

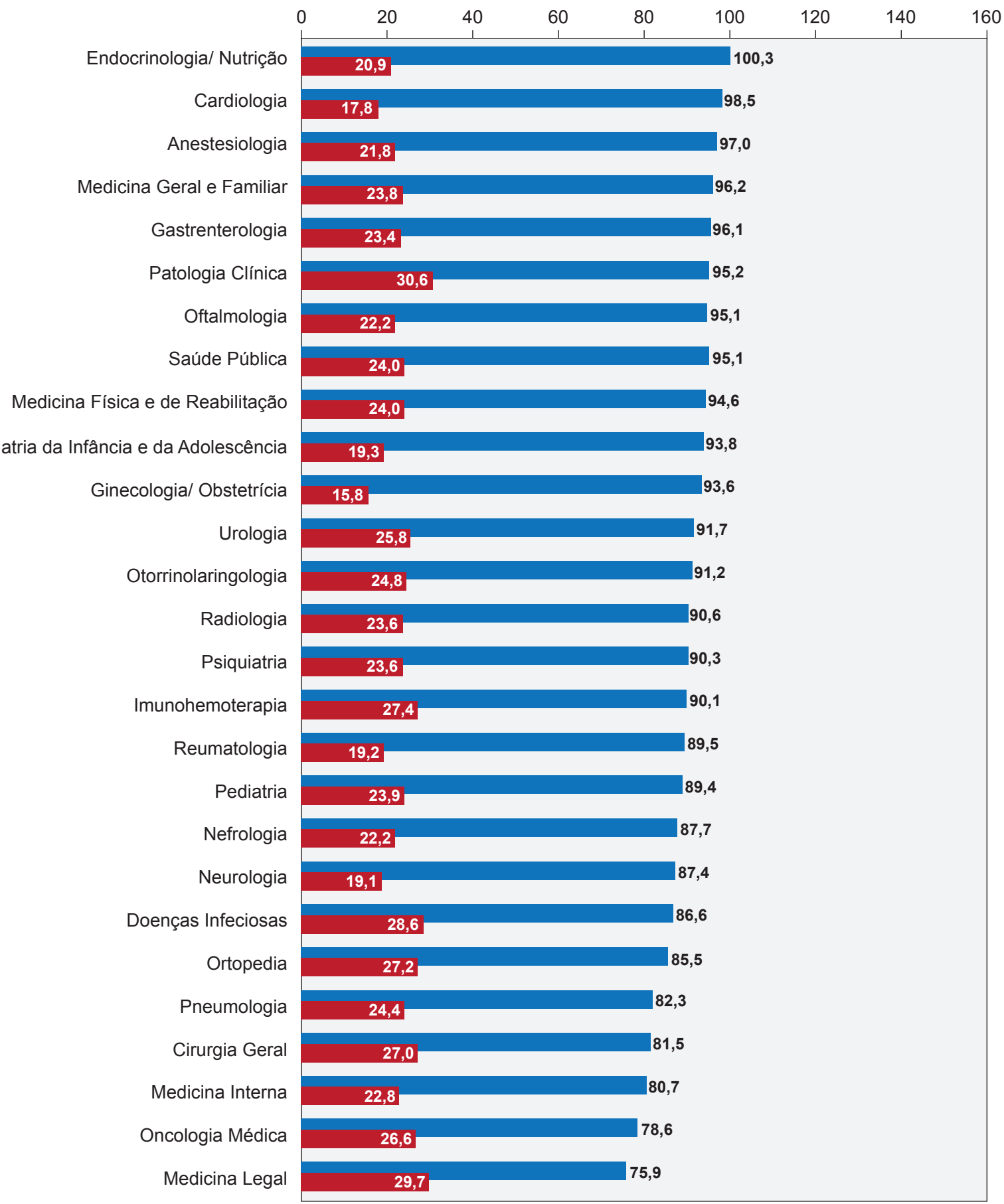


Relativamente à tipologia de instituição hospitalar (Tabela 5D), salienta-se que os médicos internos de instituições de tipologia I apresentam o grau de satisfação mais elevado e são simultaneamente os médicos internos mais satisfeitos com a Direção do Internato Médico. Sugere-se como hipótese para este resultado a possível maior proximidade dos médicos internos com a sua Direção do Internato Médico nestas instituições, a qual se poderá traduzir num maior apoio e facilidade de resolução de problemas. O menor número de médicos internos existentes nestas instituições poderá contribuir ainda, em particular em especialidades nas quais sejam realizados procedimentos interventivos, para uma maior possibilidade de aprendizagem e consequentemente maior satisfação dos médicos internos. Apesar de não ter sido avaliado no presente inquérito, é possível que a perceção de maior probabilidade de contratação por estas instituições de formação após a conclusão do Internato Médico contribua também para a maior satisfação detetada. No estudo realizado em 2014 os médicos internos de hospitais centrais obtiveram grau de satisfação inferior, dado coincidente com os resultados do presente estudo. Os autores colocaram como hipóteses para este resultado, a menor componente pessoal, a maior competição e a maior intensidade, frequência e exigência do trabalho em serviço de urgência nestas instituições. ${ }^{12}$

Entre 2011 e 2013 foi realizado um inquérito de satisfação dirigido a médicos internos de MGF da região Norte. Este estudo concluiu que, apesar dos médicos internos se encontrarem satisfeitos com o seu programa de Formação Específica, estariam insatisfeitos com o tempo despendido em atividades não clínicas para efeitos curriculares. ${ }^{35}$ No presente estudo verificou-se que os médicos internos consideraram ser contactados telefonicamente de modo inapropriado e não existir um programa educacional relevante para as suas necessidades, aspetos que poderão estar relacionados com os resultados do estudo prévio. De destacar ainda que, no presente estudo, a especialidade de MGF apresentou um dos mais elevados graus de satisfação.

O último estudo de âmbito nacional sobre a satisfação com o Internato Médico em Portugal, datado de 2014, obteve 804 respostas, correspondendo a uma taxa de resposta de $12,25 \%$. Os autores concluíram que apesar dos médicos internos apresentarem níveis positivos de satisfação com a sua profissão, a maioria considerava existir um agravamento significativo das condições para a prática clínica em Portugal, o que poderia justificar a percentagem elevada de médicos que consideravam emigrar no final do seu Internato Médico. De destacar que o referido estudo não incluiu médicos internos do Ano Comum e não avaliou os motivos da satisfação dos médicos internos. O presente estudo, tendo sido realizado com um questionário desenvolvido especificamente para médicos Internos, permite identificar quais os aspetos com os quais os médicos internos estão mais insatisfeitos, de entre os analisados. De salientar que em ambos os estudos, a especialidade na qual os médicos internos se encontravam menos satisfeitos foi Medicina Legal, o que deverá ser alvo de análise pelas entidades que

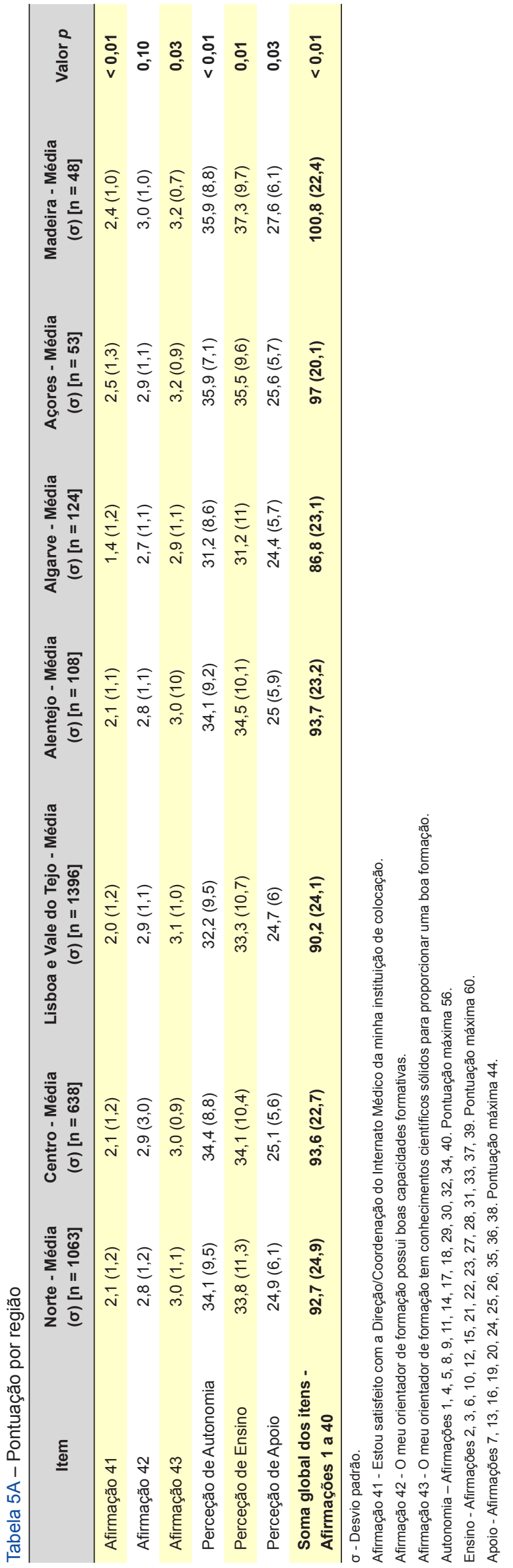




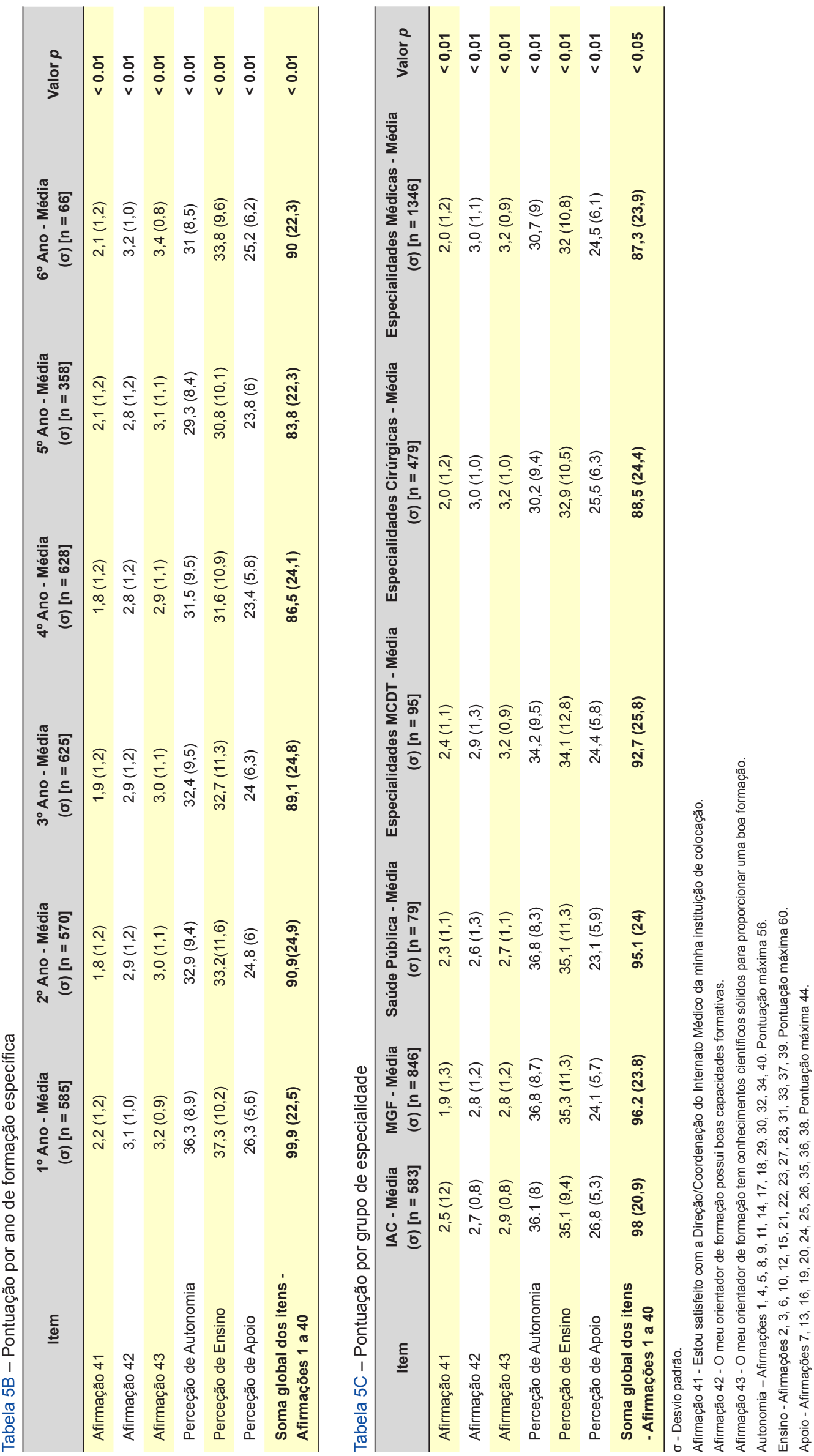


Tabela 5D - Pontuação por tipologia de Instituição Hospitalar

\begin{tabular}{|c|c|c|c|c|c|}
\hline Item & $\begin{array}{l}\text { Grupo I- Média } \\
(\sigma)[n=842]\end{array}$ & $\begin{array}{l}\text { Grupo II- Média } \\
(\sigma)[n=537]\end{array}$ & 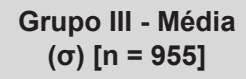 & $\begin{array}{l}\text { Grupo IV - Média } \\
\qquad(\sigma)[n=86]\end{array}$ & Valor $p$ \\
\hline Afirmação 41 & $2,3(1,2)$ & $2,0(1,2)$ & $2,0(1,1)$ & $2,1(1,3)$ & $<0,01$ \\
\hline Afirmação 42 & $2,9(1,0)$ & $3(1,0)$ & $2,9(1,1)$ & $3,1(1,0)$ & 0,07 \\
\hline Afirmação 43 & $3,1(0,9)$ & $3,1(1,0)$ & $3,2(1,0)$ & $3,3(0,8)$ & 0,05 \\
\hline Perceção de Autonomia & $34,1(9)$ & $32,3(9,2)$ & $30,3(8,8)$ & $31,4(9,9)$ & $<0,01$ \\
\hline Perceção de Ensino & $34,6(10,4)$ & $33,9(10,1)$ & $31,2(10,4)$ & $33,3(11,4)$ & $<0,01$ \\
\hline Perceção de Apoio & $26,1(5,9)$ & $25,5(5,6)$ & $24,3(5,9)$ & $24,6(6,1)$ & $<0,01$ \\
\hline $\begin{array}{l}\text { Soma global dos itens - } \\
\text { Afirmações } 1 \text { a } 40\end{array}$ & $94,7(23,4)$ & $91,7(23,1)$ & $85,7(22,9)$ & $89.3(25.6)$ & $<0,01$ \\
\hline
\end{tabular}

$\sigma$ - Desvio padrão.

Afirmação 41 - Estou satisfeito com a Direção/Coordenação do Internato Médico da minha instituição de colocação.

Afirmação 42 - O meu orientador de formação possui boas capacidades formativas.

Afirmação 43 - O meu orientador de formação tem conhecimentos científicos sólidos para proporcionar uma boa formação.

Autonomia - Afirmações 1, 4, 5, 8, 9, 11, 14, 17, 18, 29, 30, 32, 34, 40. Pontuação máxima 56.

Ensino - Afirmações 2, 3, 6, 10, 12, 15, 21, 22, 23, 27, 28, 31, 33, 37, 39. Pontuação máxima 60.

Apoio - Afirmações 7, 13, 16, 19, 20, 24, 25, 26, 35, 36, 38. Pontuação máxima 44.

regulamentam a especialidade. ${ }^{12}$ Apresenta-se como hipótese para as especialidades de Medicina Interna e Cirurgia Geral se encontrarem entre as especialidades com menor grau de satisfação, o desgaste associado a estas especialidades, em particular no que diz respeito à sobrecarga de trabalho em serviço de urgência.

Destaca-se ainda que a população de médicos internos é mutável e que desde a realização do referido estudo foram implementadas diversas alterações no Internato Médico em Portugal, incluindo a alteração do Regulamento do Internato Médico e a discussão e publicação do novo Regulamento do Internato Médico e Serviço de Urgência. ${ }^{12,7}$ Neste sentido, a realização regular de inquéritos de satisfação é desejável de modo a que possa ser aferido o impacto de medidas tomadas, à semelhança da realidade existente há vários anos noutros países. ${ }^{18,19}$

Uma revisão sistemática publicada em 2016 sobre a aplicação do questionário PHEEM em instituições de diferentes países apresentou as pontuações globais médias de satisfação com o internato, existindo estudos quer com resultados superiores quer com resultados inferiores aos obtidos no presente estudo. ${ }^{26}$ Destacamos que as cinco afirmações que obtiveram pontuação mais baixa no presente estudo, correspondem a afirmações frequentemente citadas como tendo obtido pontuação mais baixa nos estudos incluídos na revisão sistemática. De realçar que não identificámos outros estudos nos quais o inquérito fosse aplicado a nível nacional. No entanto, num dos estudos incluídos nesta revisão sistemática, é possível identificar a pontuação global média dos internos em fase de formação equivalente ao Ano Comum em instituições do Reino Unido e do Chile. Neste contexto, os internos de Ano Comum em Portugal apresentaram pontuação global média igual à dos internos do Chile (98) e inferior à dos internos do Reino Unido (113). ${ }^{36}$

O inquérito foi realizado no segundo trimestre, dado ter sido considerado que a realização após o primeiro trimestre permitiria por um lado que os médicos internos que tivessem iniciado o Internato Médico em 2016 já tivessem constituído opinião crítica sobre a realização de Internato Médico na sua instituição e por outro evitar o primeiro trimestre, o qual constitui o período do ano mais frequente de avaliações anuais dos médicos internos e de preparação para o exame final de especialidade, o que condicionaria as respostas, pela ansiedade gerada nos médicos internos. Permitiu ainda evitar realizar o inquérito nos meses de Verão, os quais poderiam encontrar-se associados a menor taxa de respostas.

Apesar dos resultados obtidos, o presente estudo apresenta várias limitações. O questionário utilizado foi originalmente desenvolvido para especialidades hospitalares. No entanto, versões traduzidas e adaptadas deste questionário já foram previamente utilizadas em especialidades extra-hospitalares em diferentes países. ${ }^{19,30}$ Não foi realizada validação da tradução e adaptação do questionário para a população portuguesa pelo que a interpretação dos resultados deve ter este facto em consideração, em particular em análises comparativas entre resultados obtidos em Portugal e resultados obtidos em instituições estrangeiras. De destacar que a disponibilização de questionário online via link individualizado para o e-mail pessoal dos médicos internos, apesar de impossibilitar a duplicação de resultados, poderia conduzir à diminuição do número de respostas e condicionamento do conteúdo das mesmas, dado não garantir o anonimato da resposta. Por este motivo, e tendo em consideração que o mesmo método foi previamente aplicado a médicos internos portugueses, optou-se pela disponibilização de questionário online via link genérico, disponibilizado online através do software Survio ${ }^{\circledR} .{ }^{35}$ Apesar de não ser possível excluir a possibilidade de existência de respostas duplicadas, $52 \%$ das respostas demoraram entre 5 e 10 minutos a ser preenchidas e $17 \%$ das respostas demoraram um período de tempo superior a 10 minutos a ser respondidas. Neste sentido, caso por qualquer motivo um interno respondesse mais do que uma vez ao questionário, teria de despender um período de tempo considerável. 
Tendo em consideração as 3456 respostas obtidas, consideramos que a eventual existência de respostas duplicadas, contribui de modo desprezível para os resultados globais do questionário. O questionário PHEEM inclui três categorias de afirmações destinadas a avaliar Ensino, Autonomia e Apoio. No entanto, não foi avaliado se as três categorias descritas se mantêm após a tradução e adaptação do questionário para a língua portuguesa, devendo a interpretação dos resultados destas categorias ter este aspeto em consideração. De salientar que cada uma destas categorias inclui um número diferente de afirmações pelo que as pontuações máximas totais são diferentes, dificultando a realização de comparações entre as três categorias. Neste sentido, a análise da pontuação obtida em cada afirmação isoladamente bem como da pontuação global obtida será mais valorizável. De realçar ainda que o inquérito foi destinado apenas a médicos internos, pelo que a interpretação dos resultados não deverá ser generalizada a médicos especialistas.

Consideramos que os resultados deste inquérito deverão ser tidos em conta na implementação de medidas dirigidas a este grupo profissional, o qual pelas suas especificidades deve ser alvo de abordagem individualizada.

\section{CONCLUSÃO}

A nível nacional os médicos internos apresentaram, na globalidade, níveis elevados de satisfação, destacando-se marcadas assimetrias de acordo com o ano de especialidade, região e grupo de especialidade. Relativamente a aspetos passiveis de melhoria, e tendo em consideração os resultados globais do questionário, sugerimos a criação de tempo de estudo definido no horário de trabalho do médico interno, com prejuízo da carga horária assistencial; a me-
Ihoria dos locais nas instituições de formação destinados ao descanso durante o trabalho em período de urgência; a criação nas instituições de formação de um manual informativo e de procedimentos para médicos internos e a criação de estruturas de apoio ao percurso profissional do médico interno e carreira futura.

Consideramos que os resultados deste inquérito deverão ser alvo de análise pelas entidades competentes que regulamentam o Internato Médico em Portugal, constituindo uma ferramenta de apoio à implementação de medidas futuras de âmbito local e nacional, visando a melhoria do Internato Médico. Consideramos que a realização regular de inquéritos de satisfação é desejável, de modo a que possa ser aferido o impacto das medidas tomadas.

\section{PROTECÇÃO DE PESSOAS E ANIMAIS}

Os autores declaram que os procedimentos seguidos estavam de acordo com os regulamentos estabelecidos pelos responsáveis da Comissão de Investigação Clínica e Ética e de acordo com a Declaração de Helsínquia da Associação Médica Mundial.

\section{CONFIDENCIALIDADE DOS DADOS}

Os autores declaram ter seguido os protocolos do seu centro de trabalho acerca da publicação de dados.

\section{CONFLITOS DE INTERESSE}

Os autores declaram não ter qualquer conflito de interesse relativamente ao presente artigo.

\section{FONTES DE FINANCIAMENTO}

Os autores declaram não ter recebido qualquer subsídio relativo ao presente artigo.

\section{REFERÊNCIAS}

1. Diogo PG, Moreira A, Coimbra A, Silva A, Martins A, Mendonça C, et al. Estudo sobre as condições pedagógicas das escolas médicas portuguesas: Uma análise nacional sobre a satisfação estudantil, rácios estudante-tutor e número de admissões. Acta Med Port. 2016;29:301-9.

2. Associação Nacional de Estudantes de Medicina. Planeamento integrado da formação médica em portugal. Lisboa: ANEM; 2016.

3. Organisation for Economic Cooperation and Development. Health at a glance: Europe 2014. Paris: OECD; 2014.

4. Santana P, Peixoto H, Loureiro A, Costa C, Nunes C, Duarte N. Estudo de evolução prospectiva de médicos no sistema nacional de saúde. Coimbra: Universidade de Coimbra; 2013.;

5. Santana P, Peixoto H, Duarte N. Demografia médica em Portugal: análise prospetiva. Acta Med Port. 2014;27:246-51.

6. Assembleia da República. Decreto-Lei n.o 86/2015 de 21 de Maio. Diário da República. 2015;98:2-11.

7. Assembleia da República. Portaria n.o 224-B/2015 de 29 de Julho. Diário da República. 2015;146:6-24.

8. Boor K, Scheele F, Van Der Vleuten CP, Scherpbier AJ, Teunissen PW, Sijtsma K. Psychometric properties of an instrument to measure the clinical learning environment. Med Educ. 2007;41:92-9.

9. Hoff TJ, Pohl H, Bartfield J. Creating a learning environment to produce competent residents: the roles of culture and context. Acad Med. 2004;79:532-9.

10. Haas JS, Cook EF, Puopolo AL, Burstin HR, Cleary PD, Brennan TA. Is the professional satisfaction of general internists associated with patient satisfaction? J Gen Intern Med. 2000;15:122-8.

11. Goddard AF. Lessons to be learned from the UK junior doctors' strike.
JAMA. 2016;1-2.

12. Martins MJ, Laíns I, Brochado B, Oliveira-Santos M, Pinto Teixeira P, Brandão M, et al. Satisfação com a especialidade entre os internos da formação específica em Portugal. Acta Med Port. 2015;28:209-21.

13. Australian Medical Association. 2010 AMA Specialist Trainee Survey. Barton: AMA; 2011.

14. Australian Council for Educational Research. AGPT Registrar Satisfaction Survey. Cranberra: GPET; 2013.

15. Kelly MO, Kelly FO, Ciardha DÓ. A national survey of GP trainees 2012. Dublin: Trinity College Centre for Health Sciences; 2012.

16. Collins C, Mansfield G, O'Ciardha D, Ryan K. Planning for the future Irish General Practitioner workforce - informed by a national survey of GP trainees and recent GP graduates. Dublin: Irish College of General Practitioners; 2014.

17. Australian Council for Educational Research. AGPT Registrar Satisfaction Survey October 2014. Cranberra: GPET; 2014.

18. Grabek J, Churchill JA, Banfield S. The AMA Specialist Trainee Survey 2014: a survey of hospital-based specialty trainees and general practice registrars. Med J Aust. 2015;203:254e-5.

19. General Medical Council. National training survey 2014 Key findings. London: GMC; 2014.

20. Gough J, Bullen M. PHEEM “Downunder.” Med Teach. 2010;32:161-3.

21. Clapham M, Wall D, Batchelor A. Educational environment in intensive care medicine--use of Postgraduate Hospital Educational Environment Measure (PHEEM). Med Teach. 2007;29:e184-91.

22. Aspegren K, Bastholt L, Bested KM, Bonnesen T, Ejlersen E, Fog I, et al. Validation of the PHEEM instrument in a Danish hospital setting. Med 
Teach. 2007;29:404-506.

23. Vieira JE. The postgraduate hospital educational environment measure (PHEEM) questionnaire identifies quality of instruction as a key factor predicting academic achievement. Clinics. 2008;63:741-6.

24. Riquelme A, Herrera C, Aranis C, Oporto J, Padilla O. Psychometric analyses and internal consistency of the PHEEM questionnaire to measure the clinical learning environment in the clerkship of a Medical School in Chile. Med Teach. 2009;31:e221-5.

25. Algaidi SA. Assessment of Educational Environment for Interns Using Postgraduate Hospital Educational Environment Measure (PHEEM). J Taibah Univ Med Sci. 2010;5:1-12.

26. Chan CY, Sum MY, Lim WS, Chew NW, Samarasekera DD, Sim K. Adoption and correlates of Postgraduate Hospital Educational Environment Measure (PHEEM) in the evaluation of learning environments - a systematic review. Med Teach. 2016;1-8.

27. Galli A, Brissón ME, Soler C, Lapresa S, De Lima AA. Assessment of educational environment in cardiology residencies. Rev Argent Cardiol. 2014;82:373-8.

28. Auret KA, Skinner L, Sinclair C, Evans S. Formal assessment of the educational environment experienced by interns placed in rural hospitals in Western Australia. Rural Remote Health. 2013;13:1-11.
29. Roff S, McAleer S, Skinner A. Development and validation of an instrument to measure the postgraduate clinical learning and teaching educational environment for hospital-based junior doctors in the UK. Med Teach. 2005;27:326-31.

30. Anderson K, Haesler E, Stubbs A, Molinari K. Comparing general practice and hospital rotations. Clin Teach. 2015;12:8-13.

31. Khoja AT, Tawfik Khoja A. Evaluation of the educational environment of the Saudi family medicine residency training program. J Fam Community Med. 2015;49:49-56.

32. Assembleia da República. Portaria n.o 82/2014 de 10 de Abril. Diário da República. 2014;71:9083-100.

33. Assembleia da República. Portaria n.o 54/2010 de 21 de Janeiro. Diário da República. 2010;14:212-6.

34. Monteiro F. Médicos recusam vagas para exercer no sul e interior do país. Expresso. 2016.

35. Azevedo A, Domingues B, Moura J, Santos L. Estão os internos satisfeitos com o internato de Medicina Geral e Familiar? Rev Port Med Geral e Fam. 2014;30:24-30.

36. Wall D, Clapham M, Riquelme A, Vieira J, Cartmill R, Aspegren K, et al. Is PHEEM a multi-dimensional instrument? An international perspective. Med Teach. 2009;31:e521-7. 